\title{
MHD Stagnation Point Flow with Heat Transfer Past a Porous Sheet along with Viscous Dissipation and Thermal Radiation
}

\author{
G. Narender ${ }^{1}$, Dr. G. Sreedhar Sarma ${ }^{2}$ and Dr. K. Govardhan ${ }^{3}$ \\ ${ }^{1}$ Asst. Professor, CVR College of Engineering / H \& S Department(Mathematics), Hyderabad, India. \\ Email: gnriimc@gmail.com \\ ${ }^{2}$ Assoc. Professor, CVR College of Engineering / H \& S Department(Mathematics), Hyderabad, India. \\ Email: sarma.sreedhar@gmail.com \\ ${ }^{3}$ Asst. Professor, GITAM University, Department of Mathematics, Hyderabad, Telangana State, India. \\ Email: govardhan_kmtm@yahoo.co.in
}

\begin{abstract}
In this article, we study the magnetohydrodynamics stagnation point flow for the upper-convected Maxwell fluid with the viscous dissipation and thermal radiation effects using the Cattaneo-Christov heat flux model. The flow equations are reconstructed and the obtained set of partial differential equations is then converted into an arrangement of nonlinear, coupled O.D.E. by utilising some reasonable similarity transformations. After this, the set of O.D.E. is solved by applying shooting method. Graphs and tables describe the behavior of physical parameters.
\end{abstract}

Index Terms: Maxwell fluid; Viscous dissipation; Thermal radiation; Magnetohydrodynamics; Shooting method.

\section{INTRODUCTION}

"The point in the flow field where the fluid's velocity is zero is called stagnation point". The study of viscous, incompressible, fluid past a permeable plate or sheet has great importance in the field of fluid dynamics. During the past few decades, the work on stagnation point flow of an incompressible fluid past a permeable sheet has got significant importance because of its large number of applications in manufacturing industries. Some of the main applications are refrigeration of electrical gadgets by fan, atomic receptacles cooling for the duration of emergency power cut, solar receiver, etc. The study of two-dimensional (2D) stagnation point flow was first investigated by Hiemenz [1], whereas for getting the accurate solution, Eckert [2] extended this problem by adding the energy equation. In view of that Mahapatra and Gupta [3], Ishak et al. [4], and Hayat et al. [5] have studied the effects of heat transfer in stagnation point over a permeable plate.

"The study of magnetic properties of electrically conducting fluids is known as Magnetohydrodynamics (MHD)". The study of MHD fluid flow was first introduced by Swedish Physicist, Alfven [6]. The effect of heat transfer in Magnetohydrodynamics flow of Jeffrey fluid model over a permeable plate is invested by Hayat et al. [7]. Mustafa et al. [8] inspected the Magnetohydrodynamics flow of Maxwell fluid with heat transfer.
The study of flow behaviour and heat transfer generated by means of stretching medium, has plenty of significance in numerous industrialized developments (e.g, in the process of rubber and plastic sheets manufacturing, upgrading the solid materials like crystal, turning fibers etc). The most widely used coolant liquid among them is water. In above cases, flow behavoiur and heat transfer investigation is of major importance because final product quality be determined to bulk level on the basis of coefficient of skin friction and heat transfer surface rate. Numerous investigators talked over different traits of stretching flow problem. Some of them are Crane [9], Chaim [10], Liao and Pop [11], Khan and Sanjayanand [12], and Fang et al. [13].

In future, advancement in nano-technology is expected for making unbelievable changes in our lives. A very big number of researchers are working in this area due to its great use in the engineering and its linked areas. In the process of air cleaning, development of microelectronics, safety of nuclear reactors etc, thermophoretic magnetohydrodynamic flow of heat and mass transfer consumes prospective uses. Choi [14] was the first who introduced the idea of "nanofluids" and presented the report on the heat transfer properties of nanofluids. The thorough explosure on thermophoretic flow was examined by Derjaguin and Yalamov [15]. Heat and mass transfer of MHD thermophoretic stream above plane surface was also studied by Issac and Chamka [16]. Thermophoresis effect on aerosol particles was investigated by Tsai [17]. In fluid temperature, no doubt, viscous dissipation produces a considerable ascend. This would happen because of change in kinetic motion of fluid into thermal energy.

Viscous dissipation is unavoidable in case of flow field in high gravitational field. Viscous flow past a nonlinearly stretching sheet was deliberated by Vajravelu [18]. For external natural convention flow over a stretching medium, the impact of viscous dissipation was also studied by Mollendro and Gebhart [19], whereas the impact of viscous dissipation and Joule heating on the forced convection flow with thermal radiation was presented by Duwairi [20]. 
Our prime objective is, we providing a review study of Shah et al. [21] and extend the flow analysis with viscous dissipation parameters.

\section{Mathematical Modeling}

Consider the time independent, incompressible, twodimensional MHD, laminar, and steady state flow of a fluid past a semi-infinite stretching surfece. The geometry of the flow model is given in Figure 1.

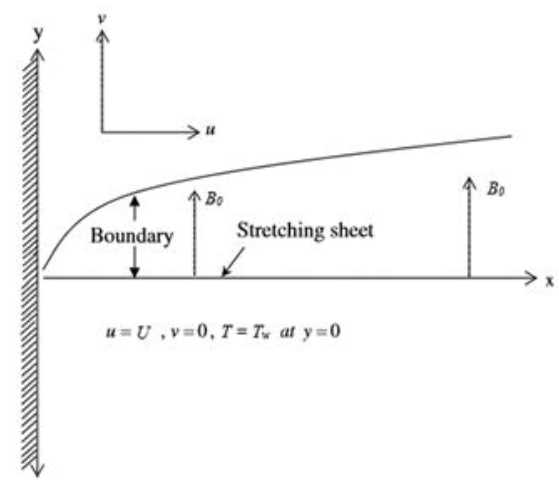

Figure 1. Geometry for the flow under consideration.

Here Cattaneo-Christov heat flux model is under consideration. Along $y$-axis, a constant magnetic field of strength $B_{0}$ is applied perpendicular to $x$-axis. Further its is supposed that the induced magnetic field is negligible. It is supposed that boundary layer approximations are appropriate to the governing equations considered by Renardy for "Maxwell fluid models". By making use of boundary layer approximations, the arrangement of representing PDEs like continuity, momentum and energy equations can be expressed as follows:

$$
\frac{\partial u}{\partial x}+\frac{\partial v}{\partial y}=0
$$

$u \frac{\partial \mathrm{u}}{\partial \mathrm{x}}+\mathrm{v} \frac{\partial \mathrm{u}}{\partial \mathrm{y}}+\lambda_{1}\left(u^{2} \frac{\partial^{2} u}{\partial x^{2}}+v^{2} \frac{\partial^{2} u}{\partial y^{2}}+2 u v \frac{\partial^{2} u}{\partial x \partial y}\right)=v \nabla^{2} u-$ $\frac{\sigma B_{0}^{2}}{\rho}(u)$

$\rho C_{p}\left(u \frac{\partial \mathrm{T}}{\partial \mathrm{x}}+\mathrm{v} \frac{\partial \mathrm{T}}{\partial \mathrm{y}}\right)=-\nabla \cdot \mathrm{q}+\sigma B_{0}^{2} u^{2}-\frac{\partial q_{r}}{\partial y}+\frac{v}{\rho c_{f}}\left(\frac{\partial u}{\partial y}\right)^{2}$

where $u$ and $v$ are the components of velocity along the $x$ and $y$ directions respectively. Moreover, $\lambda_{1}$ denotes the relaxation time, $\rho$ denotes the fluid's density, $B_{0}$ is constant magnetic field, $\sigma$ be the electric conductivity constant, kinematic viscosity is denoted by $v, C_{p}$ is the specific heat, fluid temperature is $T, q_{r}$ is the radiative heat flux. According to Christov, we have $q+\lambda_{2}\left(\frac{\partial q}{\partial t}+V \cdot \nabla q+(\nabla \cdot V) q\right)=-k \nabla T$

On abolishing $q$ from Eqs. (3) and (4), we have

$$
\left.\begin{array}{l}
\left(u \frac{\partial \mathrm{T}}{\partial \mathrm{x}}+\mathrm{v} \frac{\partial \mathrm{T}}{\partial \mathrm{y}}\right) \\
+\lambda_{2}\left(\begin{array}{c}
\left(u \frac{\partial u}{\partial x}+v \frac{\partial u}{\partial y}\right) \frac{\partial T}{\partial x}+\left(u \frac{\partial v}{\partial x}+v \frac{\partial v}{\partial y}\right) \frac{\partial T}{\partial y}+ \\
u^{2} \frac{\partial^{2} u}{\partial x^{2}}+v^{2} \frac{\partial^{2} u}{\partial y^{2}}+2 u v \frac{\partial^{2} T}{\partial x \partial y}
\end{array}\right) \\
=\alpha \frac{\partial^{2} T}{\partial y^{2}}+\sigma \frac{B_{0}^{2}}{\rho c_{p}} u^{2}-\frac{1}{\rho c_{p}} \frac{\partial q_{r}}{\partial y}+\frac{v}{\rho c_{f}}\left(\frac{\partial u}{\partial y}\right)^{2}
\end{array}\right)
$$

where $V$ denotes the fluid velocity, $\lambda_{2}$ is the relaxation time and thermal diffusivity is denoted by $\alpha$. Also, the radiative heat flux $q_{r}$, by using the Rosseland approximation for radiation, can be written as

$q_{r}=\frac{-4 \sigma^{*}}{3 k^{*}} \frac{\partial T^{4}}{\partial y}$

where $\sigma^{*}$ and $k^{*}$ stand for the Stefan-Boltzmann constant and coefficient of mean absorption.

"Expansion of $T^{4}$ about $T_{\infty}$ by making use of Taylor's series is":

$T^{4}=T_{\infty}^{4}+\frac{4 T_{\infty}^{3}}{1 !}\left(T-T_{\infty}\right)^{1}+\frac{12 T_{\infty}^{2}}{2 !}\left(T-T_{\infty}\right)^{2}+\frac{24 T_{\infty}}{3 !}(T-$

$\left.T_{\infty}\right)^{3}+\frac{24}{4 !}\left(T-T_{\infty}\right)^{4}$

Disregarding the higher order terms,

$T^{4}=T_{\infty}^{4}+4 T_{\infty}^{3}\left(T-T_{\infty}\right)$

$\Rightarrow \frac{\partial T^{4}}{\partial y}=4 T^{3} \frac{\partial T}{\partial y}$

Using (8) in (6) and the differentiate w.r.t. $y$, we get

$\frac{\partial q_{r}}{\partial y}=-\frac{16 \sigma^{*} T_{\infty}^{3}}{3 \kappa^{*}} \frac{\partial^{2} T}{\partial y^{2}}$

The boundary conditions for the above system of PDE are

$\left\{\begin{array}{c}u=U, \quad v=0, \quad T=T_{w}(x), \text { at } y=0 \\ u \rightarrow 0, \quad T \rightarrow T_{\infty}, \text { as } y=\infty\end{array}\right\}$

\section{Dimensionless Form OF THE MOdel}

Now, we introduce similarity transformations or (dimensionless variables) Shah et al. [21] which are useful in transforming the PDEs Eqs. (1) - (3) into the ODEs along with the boundary conditions Eqs. (8).

$$
\begin{array}{ll}
\eta=\sqrt{\frac{U}{v x}}(y), \quad & \theta(\eta)=\frac{T-T_{\infty}}{T_{w}-T_{\infty}}, \\
u=U f^{\prime}(\eta), & v=-\frac{1}{2} \sqrt{\frac{U v}{x}}\left(f-\eta f^{\prime}\right)
\end{array}
$$

where the prime represents derivative w.r.t $\eta, T_{\infty}$ and $T_{w}$ are the ambient and constant fluid temperature at wall respectively and $\theta$ is the dimensionless temperature. The set of corresponding ODEs is: 
$f^{\prime \prime \prime}+\frac{1}{2} f f^{\prime \prime}-\frac{\beta}{2}\left(\eta f^{\prime 2} f^{\prime \prime}+2 f f^{\prime} f^{\prime \prime}+f^{2} f^{\prime \prime \prime}\right)-M f^{\prime}=0$

$\frac{1}{P r}\left(1+\frac{4}{3} R\right) \theta^{\prime \prime}+\frac{1}{2} f \theta^{1}-\frac{\gamma}{2}\left(3 f f^{\prime} \theta^{\prime}+f^{2} \theta^{\prime \prime}\right)+M E c f^{\prime 2}=0$

The boundary conditions for the governing ODEs are

$f(\eta)=0, f^{\prime}(\eta)=1, \theta(\eta)=1$, at $\eta=0$.

$f^{\prime}(\eta) \longrightarrow 0, \theta(\eta) \rightarrow 1$, at $\eta=\infty$.

In Eqs. (12) - (13), $\beta$ is the Deborah number, $\operatorname{Pr}$ is the Prandtl number, $M$ is the magnetic parameter, radiational parameter is $R, E C$ is the Eckert number and $\gamma$ is the non-dimensional thermal relaxation time parameter. Some important dimensionless parameters are formulated as

$$
\begin{aligned}
& \beta=\frac{\lambda_{1}}{2 x}, \operatorname{Pr}=\frac{v}{\alpha}=\frac{\mu C_{p}}{k}, M=\sigma \frac{B_{0}^{2} x}{\rho U}, \quad R=\frac{4 \sigma^{*} T_{\infty}^{3}}{k \kappa^{*}}, \\
& E c=\frac{U^{2}}{C_{p}\left(T_{w}-T_{\infty}\right)} \text { and } \gamma=\frac{\lambda_{2} U}{2 x}
\end{aligned}
$$

\section{NUMERICAL SOLUTION}

As system of Eqs. (12) - (15) with the associated boundary conditions is coupled and nonlinear, so approximate solution cannot be found directly. For this we use the numerical technique i.e., the shooting method along with Adams-Moultan method. By making use of this technique, we convert the system of higher order ODEs into the system of first order ODEs.

$$
\begin{aligned}
& f^{\prime \prime \prime}=\frac{1}{2-\beta f^{2}}\left(\eta \beta f^{\prime 2} f^{\prime \prime}+2 \beta 2 f f^{\prime} f^{\prime \prime}-f f^{11}+2 M f^{\prime}\right) \\
& \theta^{\prime \prime}=\frac{3 P r}{6+8 R-3 P r \gamma f^{2}}\left(3 \gamma f f^{\prime} \theta^{\prime}-f \theta^{1}-2 M E c f^{\prime 2}-E c f^{\prime \prime 2}\right)
\end{aligned}
$$

subject to boundary conditions

$$
\begin{aligned}
& f^{\prime}(\eta)=1, f(\eta)=0 \text { at } \eta=0, f^{\prime}(\infty) \rightarrow 0 \text {, as } \eta \rightarrow \infty, \\
& \theta(\eta)=1 \text { at } \eta=0 ; \theta(\eta) \rightarrow 0, \text { as } \eta \rightarrow \infty
\end{aligned}
$$

Let us use the notations

$$
f=y_{1}, \theta=y_{4}
$$

Further denote

$f^{\prime}=y_{1}^{\prime}=y_{2}, f^{\prime \prime}=y_{2}^{\prime}=y_{3}, \theta^{\prime}=y_{4}^{\prime}=y_{5}, \theta^{\prime \prime}=y_{5}^{\prime}$.

The system of first Order ODEs along with the boundary conditions becomes

$$
\begin{array}{ll}
y_{1}^{\prime}=y_{2}, & y_{1}(0)=0 \\
y_{2}^{\prime}=y_{3}, & y_{2}(0)=1
\end{array}
$$

$$
\begin{gathered}
y_{3}^{\prime}=\frac{1}{2-\beta y_{1}^{2}}\left(\eta \beta y_{2}^{2} y_{3}+2 \beta y_{1} y_{2} y_{3}-y_{1} y_{3}+2 M y_{2}\right) \\
y_{3}(0)=s \\
y_{4}(0)=1 \\
y_{4}^{\prime}=y_{5}, \quad \frac{3 P r}{6+8 R-3 P r \gamma y_{1}^{2}}\left(3 \gamma y_{1} y_{2} y_{5}-y_{1} y_{5}-2 M E c y_{2}^{2}-E c y_{3}^{2}\right), \\
y_{5}(0)=t
\end{gathered}
$$

For solving above system numerically, we replace the domain $[0, \infty]$, by the bounded domain $\left[0, \eta_{\infty}\right]$ where $\eta_{\infty}$ is some suitable real number. In the above system of equations we have $y_{3}(\eta)$ and $y_{5}(\eta)$ at $\eta=0$ i.e., $s$ and $t$ are missing conditions and are to be chosen such that $y_{2}\left(\eta_{\infty}, s, t\right) \approx 0$ and $y_{4}\left(\eta_{\infty}, s, t\right) \approx 0$.

Finally, the choice of $\eta_{\max }=16$ was more than enough for end condition. The convergence criteria is choosen to be successive value agree up tp 2 significant digites.

\section{RESUlt AND DiscusSiON}

This section aims to investigate the numerical impacts of different parameters such as Prandtl number $\mathrm{Pr}$, nondimensional thermal relaxation time parameter $\gamma$, Deborah numbers $\beta$, Eckert number $E c$, magnetic parameter $M$ and radiational parameter $R$ displayed graphically and tablularly. The computations are worked out for different values of the effects of magnetic parameter $M$, Eckert number $E c$, Prandtl number $P r$, Deborah number $\beta$ and non dimensional thermal relaxation time parameter $\gamma$ and also discussed the effects of various physical parameters on velocity and temperature profiles.

The impact of various parameters like, Magnetic parameter, Radiational parameter, Eckert number, Prandtl number, radiational parameter is discussed graphically. In Table 1 and 2 numerical values for temperature gradient $-\theta^{\prime}(0)$ and velocity $-f^{\prime \prime}(0)$ are calculated for different physical parameters.

For visualizing the effects of different parameters on velocity $f^{\prime}(\eta)$ and temperature profile $\theta(\eta)$, graphs are plotted below. In every one of these estimations, we have considered $\gamma=$ $0.5, \operatorname{Pr}=0.72, M=0.1, \beta=0.5, R=0.23$ and $\quad E c=0.1$. Figure 2 determines the impact of magnetic parameter $M$ on dimensionless velocity $f^{\prime}(\eta)$. The graphical demonstration shows that for the increasing values of magnetic parameter $M$, there is decrease in the velocity profile. It happens for the reason that Lorentz force which decreases the horizontal flow risen by rising the magnetic parameter $M$. Figure 3 is the graphical representation which shows the temperature profile for the various values of magnetic parameter $M$. By this graph, it is observed that the effect of magnetic parameter $M$ on velocity and temperature profile is opposite. From Figure 4, it can be seen that by increasing the value of Eckret number $E c$, temperature profile also increases. The effect of radiational 
parameter $R$ on dimensionless temperature $\theta(\eta)$ is represented in Figure 5. In this graph it is observed that on increasing the value of radiational parameter $R$, temperature profile $\theta(\eta)$ also increases. So, the rate of heat transfer decreases with increase in radiational parameter $R$, and because of which temperature profile increases. In Figure 6, the influence of non-dimensional thermal relaxation time parameter $\gamma$ on temperature profile $\theta(\eta)$ is shown. This graph represents that on increasing the nondimensional thermal relaxation time parameter $\gamma$, value of temperature profile $\theta(\eta)$ decreases, because of this fact that when non dimensional thermal relaxation time parameter increases results decreases in time of deformation which causes the decrease in temperature of fluid. Figure 7 shows the influence of Deborah number $\beta$ on velocity profile $f^{\prime}(\eta)$. For the increasing values of Deborah number $\beta$, velocity increases near the plate while in the rest portion of the boundary layer it diminishes for expanding $\beta$. From Figure 8 , it can be seen that by the increase in Deborah number $\beta$, temperature profile $\theta(\eta)$ increases. Figure 9 illustrates the difference of temperature $\theta(\eta)$ for different values of the Prandtl number Pr. It is perceived that the temperature decreases, for the increasing values of Prandtl number. Decrease in thermal boundary layer comes across when $\mathrm{Pr}$ is larger and decrease in the thermal diffusivity causes rise in the Prandtl number. In this way increment in $\operatorname{Pr}$ diminishes diffusivity and the variety in thermal characteristics increments.

TABLE I.

Values Of The Reduced Nusselt Number $-\theta^{\prime}(0)$, For DifFerent VAlues of $P r, \gamma, \beta, M, E c$ and $R$.

\begin{tabular}{|c|c|c|c|c|c|c|}
\hline$P r$ & $\gamma$ & $\boldsymbol{\beta}$ & $M$ & $E c$ & $R$ & $-\boldsymbol{\theta}^{\prime}(\mathbf{0})$ \\
\hline 0.72 & 0.5 & 0.5 & 0.1 & 0.1 & 0.23 & 0.20963440 \\
\hline 0.3 & & & & & & 0.10691930 \\
\hline 0.5 & & & & & & 0.15225160 \\
\hline \multirow[t]{16}{*}{0.7} & & & & & & 0.20440960 \\
\hline & 0.2 & & & & & 0.21996200 \\
\hline & 0.3 & & & & & 0.21646850 \\
\hline & 0.4 & & & & & 0.21303090 \\
\hline & & 0.2 & & & & 0.22867970 \\
\hline & & 0.5 & & & & 0.20963430 \\
\hline & & 0.7 & & & & 0.20664860 \\
\hline & & & 0.3 & & & 0.17987930 \\
\hline & & & 0.5 & & & 0.15613890 \\
\hline & & & 0.7 & & & 0.13709520 \\
\hline & & & & 0.5 & & 0.13016200 \\
\hline & & & & 0.9 & & 0.05068963 \\
\hline & & & & 1.1 & & 0.01095345 \\
\hline & & & & & 0.3 & 0.19704990 \\
\hline & & & & & 0.7 & 0.14891000 \\
\hline & & & & & 1.8 & 0.10241040 \\
\hline
\end{tabular}

TABLE II.

COMPARISON OF $-f^{\prime \prime}(o)$ WHEN $P r=0.72, \gamma=0: 5, E c=0: 1$ and $R=0.1$.

\begin{tabular}{cccccccc}
$\boldsymbol{P r}$ & $\boldsymbol{\gamma}$ & $\boldsymbol{\beta}$ & $\boldsymbol{M}$ & $\boldsymbol{E c}$ & $\boldsymbol{R}$ & \multicolumn{2}{c}{$-\boldsymbol{f}^{\prime \prime}(\boldsymbol{o})$} \\
$\mathbf{0 . 7 2}$ & 0.5 & 0.2 & 0.1 & 0.1 & 0.23 & 0.51593330 & 0.5169288 \\
& & 0.5 & & & & 0.48199610 & 0.4822495 \\
& 0.7 & & & & 0.45818500 & 0.45824237 \\
& & & 0.1 & & & 0.48199610 & 0.4822495 \\
& & & 0.3 & & & 0.64494780 & 0.6450524 \\
& & & 0.5 & & & 0.78028870 & 0.7803249 \\
& & & 0.7 & & & 0.89726330 & 0.8972758
\end{tabular}




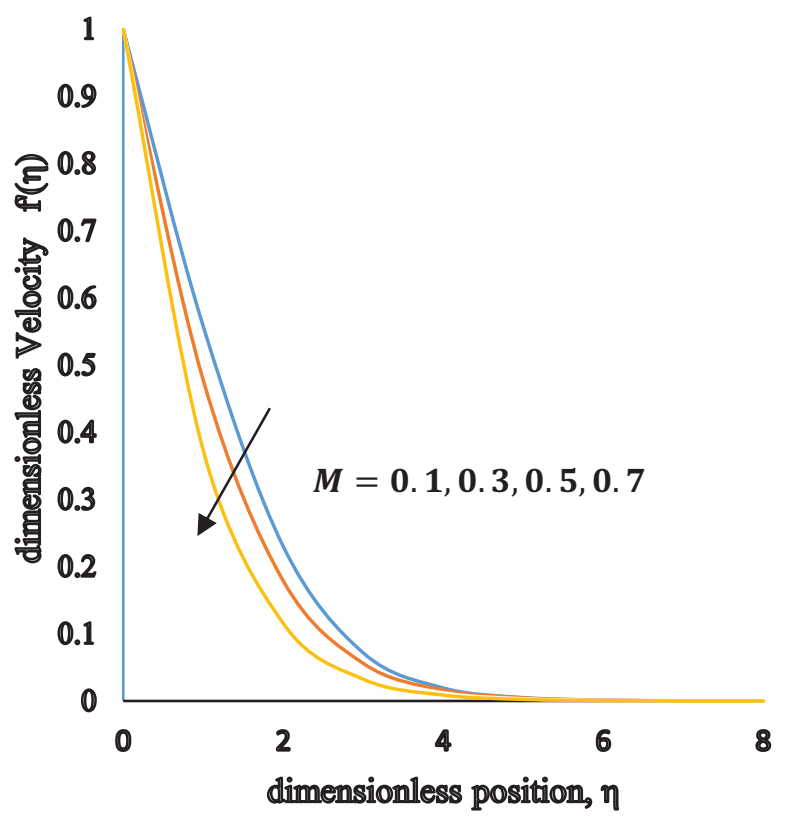

Figure 2. Dimensionless Velocity vs $M$

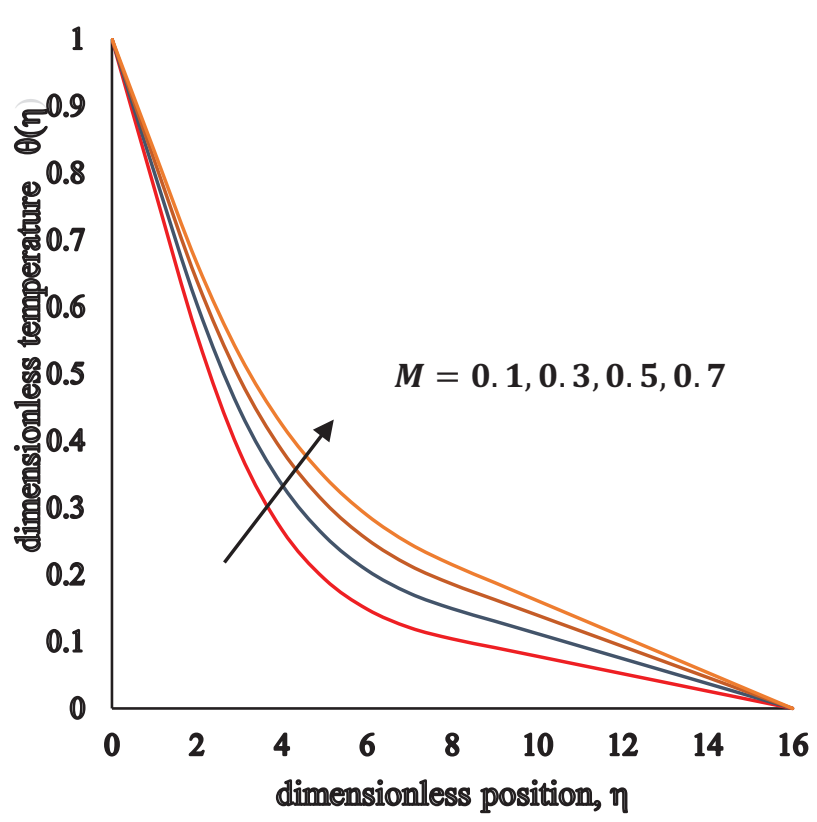

Figure 3. Dimensionless Temperature vs $M$

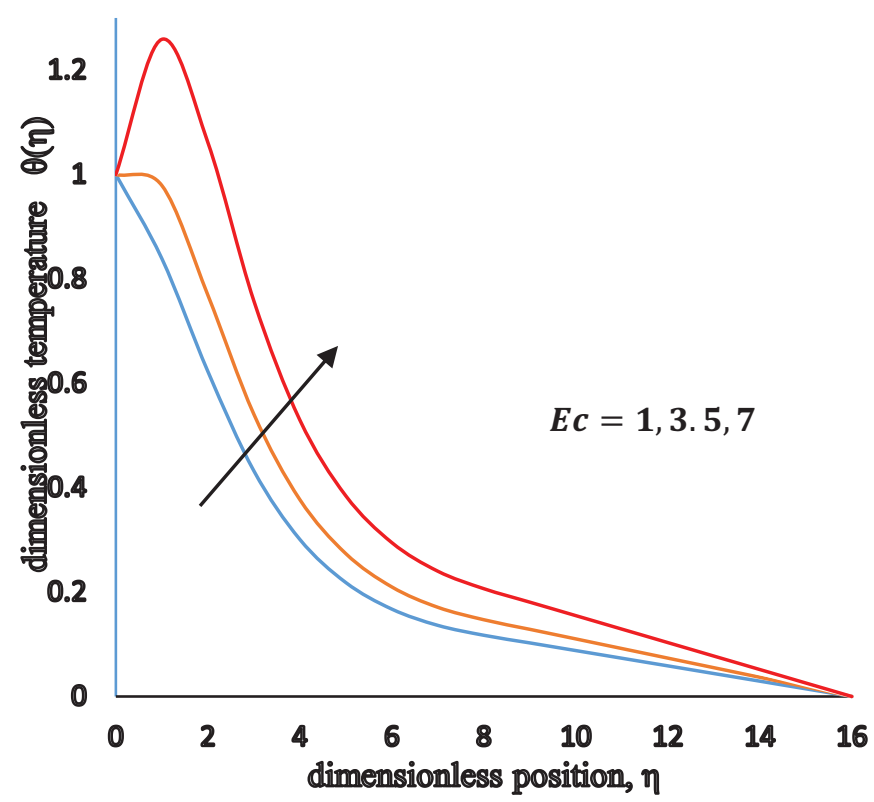

Figure 4. Dimensionless Temperature vs $E c$

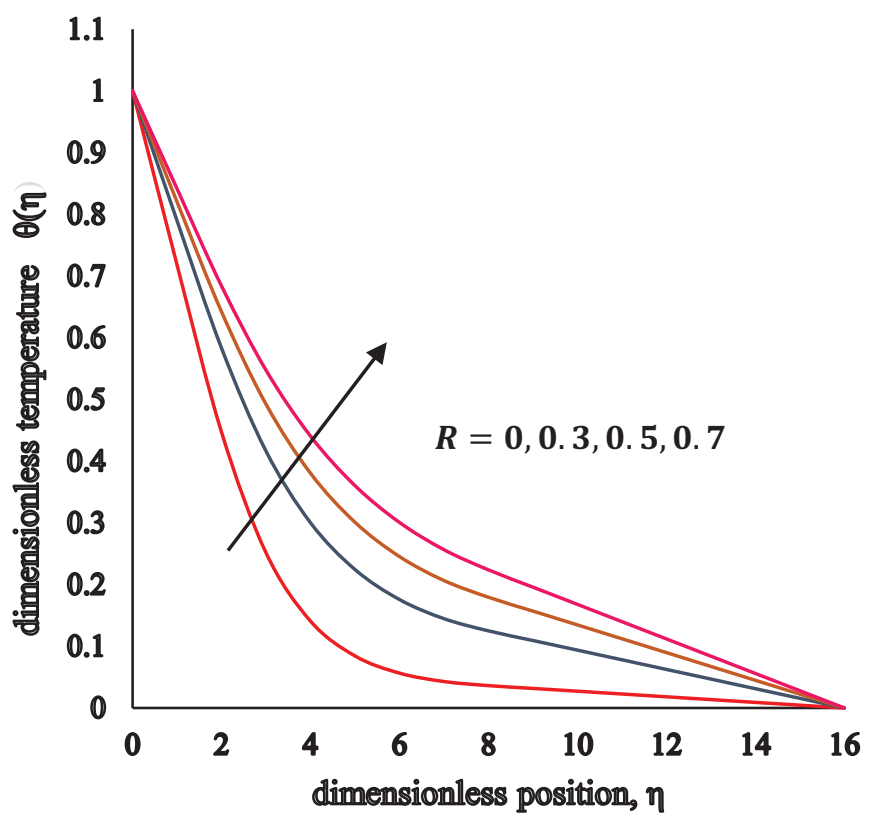

Figure 5. Dimensionless Temperature vs $R$ 


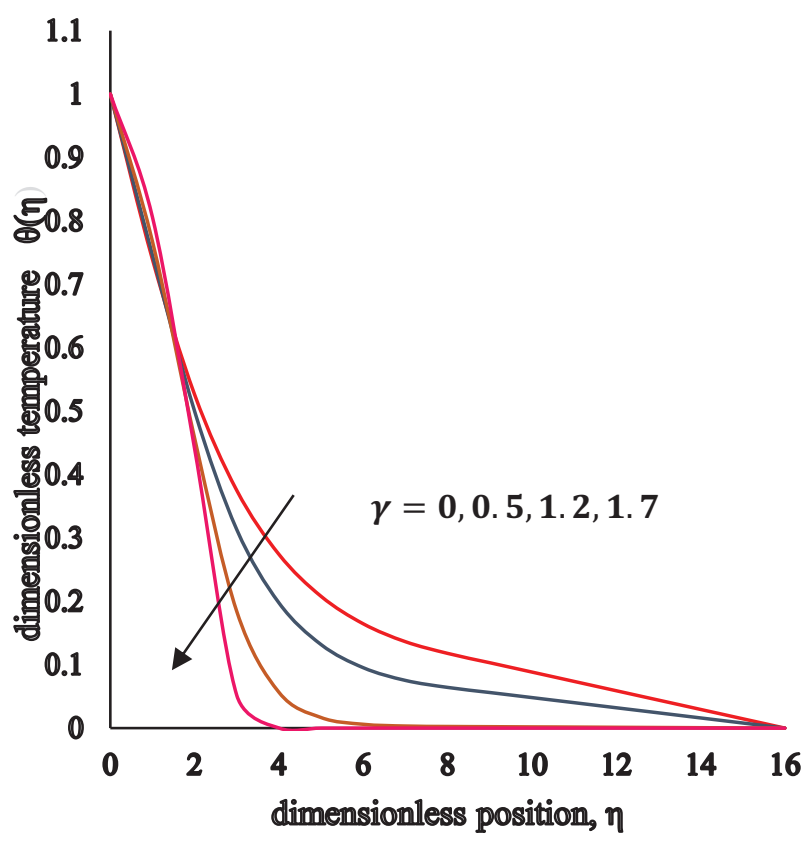

Figure 6. Dimensionless Temperature vs $\gamma$

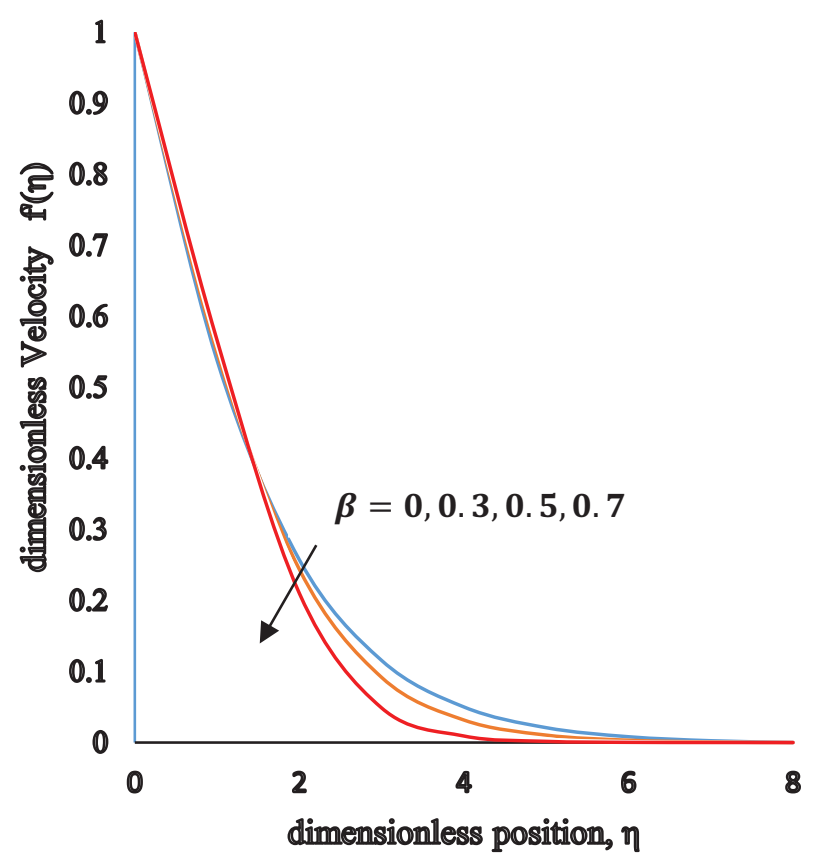

Figure 7. Dimensionless Velocity vs $\beta$

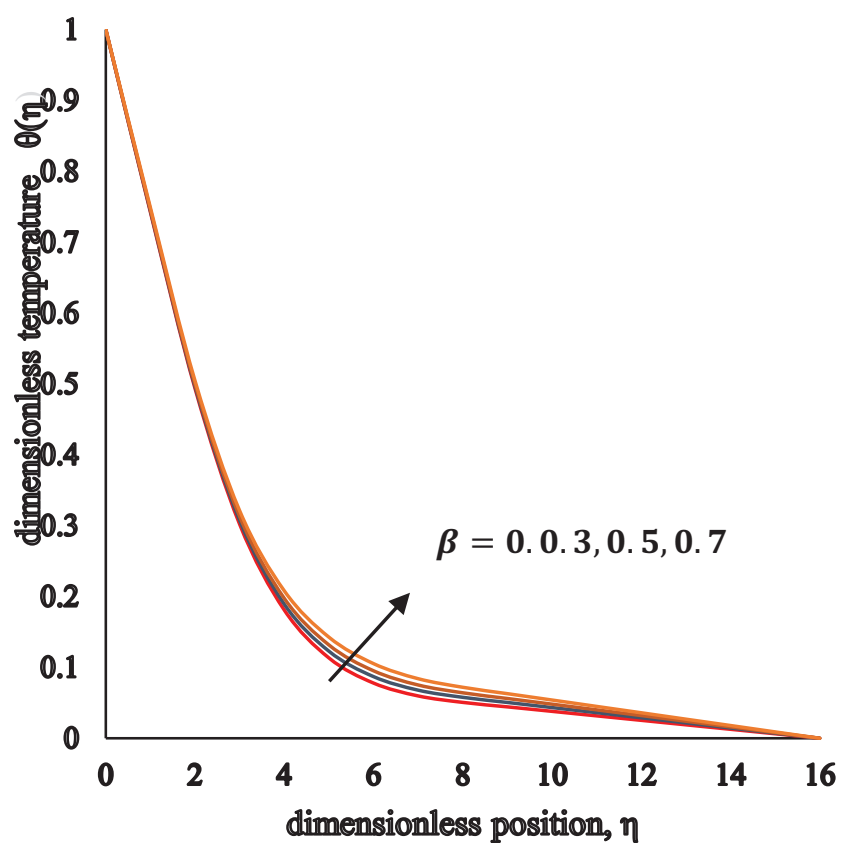

Figure 8. Dimensionless Temperature vs $\beta$

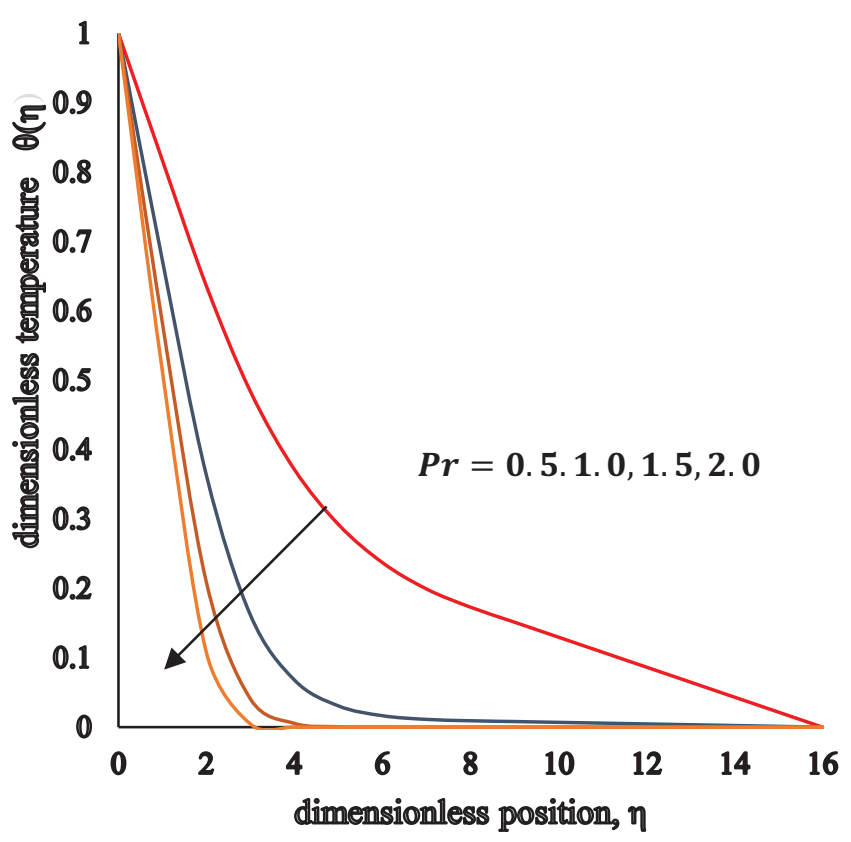

Figure 9. Dimensionless Temperature vs $\mathrm{Pr}$ 


\section{CONCLUSIONS}

Conclusions which are obtained:

- Because of strong Magnetic parameter $M$ it causes diminish in velocity and increment in temperature.

- Increase in Deborah number $\beta$ temperature increases, while the velocity decreases in the horizontal direction.

- Temperature profile rises while extending the radiation parameter and a same effect of Eckert number is seen on the temperature field.

- On temperature profile Prandtl number has decreasing effects.

- Velocity filed $f^{\prime}$ decreases for increasing values of $\beta$.

\section{REFERENCES}

[1] K. Hiemenz. Die Grenzschicht an einem in den gleichformigen Flussigkeitsstrom eingetauchten geraden Kreiszylinder. PhD thesis, 1911.

[2] E. Eckert. Die Berechnung des Warmeubergangs in der laminaren Grenzschicht mstromter Korper. VDIForschunhsheft, 1942.

[3] T. R. Mahapatra and A. S. Gupta. Heat transfer in stagnationpoint flow towards a stretching sheet. Heat and Mass transfer, 38(6):517-521, 2002.

[4] A. Ishak, R. Nazar, and I. Pop. Mixed convection boundary layers in the stagnation-point flow towards a stretching vertical sheet. Meccanica, 41(5):509-518, 2006.

[5] T. Hayat, M. Mustafa, S. A. Shehzad, and S. Obaidat. Melting heat transfer in the stagnation-point flow of an upper-convected Maxwell UCM fluid past a stretching sheet. Int. J. Numerical Methods in Fluids, 68(2):233-243, 2012.

[6] H. Alfven. Existence of electromagnetic-hydrodynamic waves. Nature, 150(3805): 405-406, 1942.

[7] T. Hayat, S. Asad, M. Mustafa, and A. Alsaedi. MHD stagnation-point flow of Jeffrey fluid over a convectively heated stretching sheet. Computers \& Fluids, 108: 179-185, 2015.

[8] M. Mustafa, J. A. Khan, T. Hayat, and A. Alsaedi. Sakiadis flow of Maxwell fluid considering magnetic field and convective boundary conditions. AIP Advances, 5 (2):027106, 2015.

[9] L. J. Crane. Flow past a stretching plate. Zeitschrift f• ur angewandte Mathematik und Physik ZAMP, 21(4):645-647, 1970.

[10] T. C. Chiam. Hydromagnetic flow over a surface stretching with a power-law velocity. Int. J. Engineering Science, 33(3):429-435, 1995.

[11] S. J. Liao and I. Pop. Explicit analytic solution for similarity boundary layer equations. Int. J. Heat and Mass Transfer, 47(1):75-85, 2004
[12] S. K. Khan and E. Sanjayanand. Viscoelastic boundary layer flow and heat transfer over an exponential stretching sheet. Int. J. Heat and Mass Transfer, 48(8):1534-1542, 2005.

[13] T. Fang, F. L. Chia-fon, and J. Zhang. The boundary layers of an unsteady incompressible stagnation-point flow with mass transfer. Int. J. Non-Linear Mechanics, 46(7):942-948, 2011.

[14] S. Choi. Enhancing thermal conductivity of fluids with nanoparticles in developments and applications of nonNewtonian flows (ed. da. siginer et hp. wang) pp.99-105, New York: Am. Soc. Mech. Eng, 1995.

[15] B. V. Derjaguin and Y. Yalamov. Theory of thermophoresis of large aerosol particles. J. Colloid science, 20(6):555-570, 1965.

[16] A. J. Chamkha and C. Issac. Effects of heat generation/absorption and thermophoresis on hydromagnetic flow with heat and mass transfer over a at surface. Int. J. Numerical Methods for Heat \& Fluid Flow, 10(4):432-449, 2000.

[17] R. Tsai. A simple approach for evaluating the effect of wall suction and thermophoresis on aerosol particle deposition from a laminar flow over a at plate. Int. C. Heat and Mass Transfer, 26(2):249-257, 1999.

[18] K. Vajravelu. Viscous flow over a nonlinearly stretching sheet. Applied mathematics and computation, 124(3):281288, 2001.

[19] B. Gebhart and J. Mollendorf. Viscous dissipation in external natural convection flows. J. Fluid mechanics, 38(01):97-107, 1969.

[20] H. M. Duwairi. Viscous and Joule heating effects on forced convection ow from radiate isothermal porous surfaces. Int. J. Numerical Methods for Heat \& Fluid Flow, 15(5):429440, 2005.

[21] S. Shah, S. Hussain, and M. Sagheer. MHD effects and heat transfer for the UCM fluid along with Joule heating and thermal radiation using Cattaneo-Christov heat flux model. AIP Advances, 6(8):085103, 2016. 